Mathematical Research Letters 5, 781-790 (1998)

\title{
A COROLLARY TO BERNSTEIN'S THEOREM AND WHITTAKER FUNCTIONALS ON THE METAPLECTIC GROUP
}

\author{
WILLIAM D. BANKS
}

In this paper, we extend and apply a remarkable theorem due to Bernstein, which was proved in a letter to Piatetski-Shapiro in the fall of 1985 [3]. The theorem is quite useful for establishing analytic continuation and rationality results in a variety of settings in the study of automorphic forms and $L$-functions, such as in the theory of local intertwining operators and local coefficients for induced representations, or in the theory of Eisenstein series. Bernstein's theorem is extraordinary in terms of the simplicity of its formulation, the elegance of its proof, and its wide range of applicability to problems requiring a proof of analytic continuation. The first section of this paper is devoted to a review of the statement and proof of Bernstein's theorem. We then prove a corollary that can be used in some situations to obtain more precise information about the location of poles. This corollary has already been applied by Friedberg and Goldberg [5] in their work on local coefficients for non-generic representations.

In the second section, we apply our corollary to Bernstein's theorem to establish the analytic continuation of the Whittaker functionals for a series of induced representations on the $n$-fold metaplectic cover of $G L_{r}(\mathbb{F})$, where $\mathbb{F}$ is a nonarchimedean local field. For the very special case of principal series, this is a result of Kazhdan and Patterson [6]. We now state our main result more precisely. Suppose that $\mathbb{F}$ contains the $n$-th roots of unity, and that $|n|_{\mathbb{F}}=1$. Let $G:=G L_{r}(\mathbb{F})$, and let $\widetilde{G}$ be the $n$-fold metaplectic cover of $G$. Let $M$ be the standard Levi subgroup in $G$ of type $\left(r_{1}, \ldots, r_{k}\right)$, and let $\widetilde{M}$ be the full metaplectic preimage of $M$ in $\widetilde{G}$. Now suppose that $\pi$ is an admissible representation of $\widetilde{M}$ with $\ell>0$ linearly independent Whittaker functionals. For every $s \in \mathbb{C}^{k}$, we can twist $\pi$ by a certain unramified quasicharacter $\delta^{s}: \widetilde{M} \rightarrow \mathbb{C}^{\times}$and then induce in the usual way to obtain a representation $\pi_{s}$ of $\widetilde{G}$. By [1], each $\pi_{s}$ also has $\ell$ linearly independent Whittaker functionals, and the main result of this section is that these functionals can be chosen to vary holomorphically with $s \in \mathbb{C}^{k}$. This result is the starting point for the general theory of local coefficients for induced representations on the metaplectic group, which should lead

Received October 9, 1998. Revised December 29, 1998. 
to interesting results on the nature of the (conjectural) Shimura correspondence from $\widetilde{G}$ to $G$.

The author wishes to thank Sol Friedberg for encouraging the publication of these results and for informing the author of the applications in [5].

\section{Bernstein's Theorem}

Let $V$ be a vector space over an arbitrary field $k$, and let $V^{*}:=\operatorname{Hom}_{k}(V, k)$, the linear dual of $V$. A subset $\Xi \subset V \times k$ is called a system of equations in $V \times k$. A solution to $\Xi$ is an element $\lambda \in V^{*}$ that satisfies $\lambda(v)=x$ for all $(v, x) \in \Xi$.

Let $\mathcal{D}$ be an algebraic variety defined over $k$, and let $k[\mathcal{D}]$ be the algebra of regular functions $\mathcal{D} \rightarrow k$. Let $V_{k[\mathcal{D}]}:=V \otimes_{k} k[\mathcal{D}]$, and for every $d \in \mathcal{D}$, let $\xi_{d}: V_{k[\mathcal{D}]} \rightarrow V$ be the unique linear map that sends $v \otimes \phi$ to $\phi(d) v$ for all $v \in V$, $\phi \in k[\mathcal{D}]$. A regular function $\mathcal{D} \rightarrow V$ is any function of the form $d \mapsto \xi_{d}(\hat{v})$ for some $\hat{v} \in V_{k[\mathcal{D}]}$.

A family of systems $\Xi_{\mathcal{D}}$ is a collection $\left\{\Xi_{d} \mid d \in \mathcal{D}\right\}$, where each $\Xi_{d}$ is a system of equations in $V \times k$ whose elements are indexed by a common indexing set $\mathcal{R}$ :

$$
\Xi_{d}=\left\{\left(v_{r, d}, x_{r, d}\right) \in V \times k \mid r \in \mathcal{R}\right\}
$$

The family $\Xi_{\mathcal{D}}$ is said to be regular if the functions $v_{r}: \mathcal{D} \rightarrow V, d \mapsto v_{r, d}$, and $x_{r}: \mathcal{D} \rightarrow k, d \mapsto x_{r, d}$, are regular for every $r \in \mathcal{R}$. In this case, we will choose for each $r \in \mathcal{R}$ an element $\hat{v}_{r} \in V_{k[\mathcal{D}]}$ such that $\xi_{d}\left(\hat{v}_{r}\right)=v_{r, d}$ for all $d \in \mathcal{D}$.

Now suppose that $V$ is a vector space over $k:=\mathbb{C}, \mathcal{D}$ an irreducible algebraic variety over $\mathbb{C}$, and $\Xi_{\mathcal{D}}$ a regular family of systems as above. Let $F:=\mathbb{C}(\mathcal{D})$, the field of fractions of $\mathbb{C}[\mathcal{D}]$, let $V_{F}:=V \otimes_{\mathbb{C}} F$, and let $V_{F}^{*}:=\operatorname{Hom}_{F}\left(V_{F}, F\right)$, the linear dual of $V_{F}$. As $\hat{v}_{r} \in V_{\mathbb{C}[\mathcal{D}]} \subset V_{F}$ and $x_{r} \in \mathbb{C}[\mathcal{D}] \subset F$ for each $r \in \mathcal{R}$, we can associate to the family $\Xi_{\mathcal{D}}$ a single system of equations $\Xi$ in $V_{F} \times F$ :

$$
\Xi:=\left\{\left(\hat{v}_{r}, x_{r}\right) \in V_{F} \times F \mid r \in \mathcal{R}\right\}
$$

If $\lambda \in V_{F}^{*}$ is a solution to $\Xi$, then $\lambda$ is $F$-linear and therefore determined by its values on elements of the form $v \otimes 1$ with $v \in V$. If $d \in \mathcal{D}$, and $\lambda(v \otimes 1)$ is defined at $d$ for all $v \in V$, then $\lambda(\hat{v})$ is defined at $d$ for every $\hat{v} \in V_{\mathbb{C}[\mathcal{D}] \text {. For such }}$ $d$, let $\lambda_{d} \in V^{*}$ be defined by $\lambda_{d}(v):=\lambda(v \otimes 1)(d)$ for all $v \in V$. It follows that $\lambda_{d}\left(\xi_{d}(\hat{v})\right)=\lambda(\hat{v})(d)$ for all $\hat{v} \in V_{\mathbb{C}[\mathcal{D}]}$. Moreover, since:

$$
\lambda_{d}\left(v_{r, d}\right)=\lambda_{d}\left(\xi_{d}\left(\hat{v}_{r}\right)\right)=\lambda\left(\hat{v}_{r}\right)(d)=x_{r}(d)=x_{r, d}, \quad \forall r \in \mathcal{R},
$$

it follows that $\lambda_{d}$ is a solution to $\Xi_{d}$.

Theorem. (Bernstein) Along with the assumptions above, suppose that $V$ has countable dimension over $\mathbb{C}$, and there exists a nonempty subset $\mathcal{D}_{0} \subset \mathcal{D}$, which 
is open in the Hausdorff topology, such that $\Xi_{d}$ has a unique solution for all $d \in \mathcal{D}_{0}$. Then $\Xi$ has a unique solution $\lambda \in V_{F}^{*}$. Moreover, on some subset $\mathcal{D}^{\dagger} \subset \mathcal{D}$, whose complement in $\mathcal{D}$ is a countable union of hypersurfaces, $\lambda_{d} \in V^{*}$ is defined and is the unique solution to $\Xi_{d}$.

Proof. To show that $\Xi$ has a solution $\lambda \in V_{F}^{*}$, we first define $\lambda$ on the $F$-linear span of $\left\{\hat{v}_{r} \mid r \in \mathcal{R}\right\}$ and then continue it arbitrarily to all of $V_{F}$. Thus, for every collection $\left\{a_{r} \in F \mid r \in \mathcal{R}\right\}$ in which only finitely many of the $a_{r}$ 's are nonzero, we define $\lambda\left(\sum a_{r} \hat{v}_{r}\right):=\sum a_{r} x_{r}$. To see that $\lambda$ is well-defined, we must show that $\sum a_{r} \hat{v}_{r}=0$ implies $\sum a_{r} x_{r}=0$. After multiplying by an appropriate nonzero element of $\mathbb{C}[\mathcal{D}]$, we can assume that every $a_{r} \in \mathbb{C}[\mathcal{D}]$. Then:

$$
\sum a_{r}(d) v_{r, d}=\xi_{d}\left(\sum a_{r} \hat{v}_{r}\right)=0, \quad \forall d \in \mathcal{D} .
$$

In particular, if $d \in \mathcal{D}_{0}$ :

$$
\left(\sum a_{r} x_{r}\right)(d)=\sum a_{r}(d) x_{r, d}=\lambda_{d}\left(\sum a_{r}(d) v_{r, d}\right)=0
$$

where $\lambda_{d} \in V^{*}$ is the unique solution to $\Xi_{d}$. Since $\sum a_{r} x_{r}$ vanishes on $\mathcal{D}_{0}$, our assumptions on $\mathcal{D}$ and $\mathcal{D}_{0}$ imply that $\sum a_{r} x_{r}=0$.

To show uniqueness, let $\left\{v_{i}\right\}$ be a countable basis for $V$, and let $\lambda \in V_{F}^{*}$ be a solution to $\Xi$. Let $\mathcal{D}_{\infty}(\lambda)$ be the union of the countable number of hypersurfaces in $\mathcal{D}$ where some $\lambda\left(v_{i} \otimes 1\right)$ has a pole. From the discussion preceding this theorem, $\lambda_{d}$ is defined and is a solution to $\Xi_{d}$ for all $d \in \mathcal{D}-\mathcal{D}_{\infty}(\lambda)$. Now suppose that $\lambda, \lambda^{\prime} \in V_{F}^{*}$ are two solutions to $\Xi$. Since $\Xi_{d}$ has a unique solution on $\mathcal{D}_{0}$, then for all $\hat{v} \in V_{\mathbb{C}[\mathcal{D}]}$ and all $d \in \mathcal{D}_{0}, d \notin \mathcal{D}_{\infty}(\lambda) \cup \mathcal{D}_{\infty}\left(\lambda^{\prime}\right)$ :

$$
\lambda(\hat{v})(d)=\lambda_{d}\left(\xi_{d}(\hat{v})\right)=\lambda_{d}^{\prime}\left(\xi_{d}(\hat{v})\right)=\lambda^{\prime}(\hat{v})(d) .
$$

From this it follows that $\lambda=\lambda^{\prime}$.

Since $\Xi$ has a unique solution $\lambda \in V_{F}^{*}$, the $F$-linear span of $\left\{\hat{v}_{r} \mid r \in \mathcal{R}\right\}$ must be all of $V_{F}$. Thus, for each $i, v_{i} \otimes 1=\sum a_{r, i} \hat{v}_{r}$ for some collection $\left\{a_{r, i} \in F \mid r \in \mathcal{R}\right\}$ in which only finitely many of the $a_{r, i}$ 's are nonzero. Let $\mathcal{D}_{\infty}^{\prime}(\lambda)$ be the union of $\mathcal{D}_{\infty}(\lambda)$ with the countable number of hypersurfaces where some $a_{r, i}$ has a pole, and let $\mathcal{D}^{\dagger}:=\mathcal{D}-\mathcal{D}_{\infty}^{\prime}(\lambda)$. If $d \in \mathcal{D}^{\dagger}, \lambda_{d}$ is defined and is a solution to $\Xi_{d}$. On the other hand, if $\lambda_{d}^{\prime}$ is any solution to $\Xi_{d}$, then $\lambda_{d}^{\prime}$ is uniquely determined on $V$ since $\left\{v_{i}\right\}$ is a basis for $V$, and:

$$
\begin{aligned}
& \lambda_{d}^{\prime}\left(v_{i}\right)=\lambda_{d}^{\prime}\left(\xi_{d}\left(v_{i} \otimes 1\right)\right)=\lambda_{d}^{\prime}\left(\xi_{d}\left(\sum a_{r, i} \hat{v}_{r}\right)\right)= \\
& \lambda_{d}^{\prime}\left(\sum a_{r, i}(d) v_{r, d}\right)=\sum a_{r, i}(d) x_{r, d} .
\end{aligned}
$$

This completes the proof. 
Corollary. Along with the assumptions of the preceding theorem, suppose that $\mathcal{D}$ is nonsingular. Let:

$$
\begin{aligned}
& \mathcal{D}^{!}:=\left\{d \in \mathcal{D} \mid \Xi_{d} \text { has a unique solution }\right\}, \\
& F^{!}:=\left\{\phi \in F \mid \phi \text { has no pole on } \mathcal{D}^{!}\right\} .
\end{aligned}
$$

If $\lambda \in V_{F}^{*}$ is the unique solution to $\Xi$, then $\lambda: V_{\mathbb{C}[\mathcal{D}]} \rightarrow F^{!}$. In particular, if $\Xi_{d}$ has a unique solution for all $d \in \mathcal{D}$, then $\lambda: V_{\mathbb{C}[\mathcal{D}]} \rightarrow \mathbb{C}[\mathcal{D}]$.

Proof. Let $\mathbb{C}^{\mathcal{D}^{!}}$be the collection of all set-theoretic functions $\mathcal{D}^{!} \rightarrow \mathbb{C}$. Our assumptions on $\mathcal{D}$ imply that $\mathbb{C}[\mathcal{D}]$ embeds in $\mathbb{C}^{\mathcal{D}^{!}}$by restriction, and that $S:=$ $\mathbb{C}[\mathcal{D}]-\{0\}$ is a multiplicative set. Let $\mathbb{C}_{\text {loc }}^{\mathcal{D}^{!}}:=S^{-1} \mathbb{C}^{\mathcal{D}^{!}}$be the localization of $\mathbb{C}^{\mathcal{D}^{!}}$ with respect to $S$, i.e., the ring of all quotients of the form $f / \alpha$ with $f \in \mathbb{C}^{\mathcal{D}^{\prime}}$ and $\alpha \in S$. Then there are natural embeddings $\mathbb{C}^{\mathcal{D}^{!}} \hookrightarrow \mathbb{C}_{\text {loc }}^{\mathcal{D}^{!}}(f \mapsto f / 1)$ and $F \hookrightarrow \mathbb{C}_{\text {loc }}^{\mathcal{D}^{!}}$(by restriction). Since $\mathcal{D}$ is nonsingular, its local rings have unique factorization, and it follows that $\mathbb{C}^{\mathcal{D}^{!}} \cap F=F^{\text {! }}$.

Let $\lambda^{!}: V_{\mathbb{C}[\mathcal{D}]} \rightarrow \mathbb{C}^{\mathcal{D}^{!}}$be defined by $\lambda^{!}(\hat{v})(d)=\lambda_{d}\left(\xi_{d}(\hat{v})\right)$ for all $\hat{v} \in V_{\mathbb{C}[\mathcal{D}]}$ and $d \in \mathcal{D}^{!}$, where $\lambda_{d}$ is the unique solution to $\Xi_{d}$. Note that $\lambda^{!}(\alpha \hat{v})=\alpha \lambda^{!}(\hat{v})$ for all $\alpha \in S$ and $\hat{v} \in V_{\mathbb{C}[\mathcal{D}]}$. We can extend $\lambda^{!}$to an $F$-linear map $\lambda_{F}^{!}: V_{F} \rightarrow \mathbb{C}_{\text {loc }}^{\mathcal{D}^{!}}$as follows. Every element in $V_{F}$ can be expressed in the form $\phi \hat{v}$ with $\phi \in F$ and $\hat{v} \in V_{\mathbb{C}[\mathcal{D}]}$. Let $\lambda_{F}^{!}(\phi \hat{v}):=\phi \lambda^{!}(\hat{v}) \in \mathbb{C}_{\text {loc }}^{\mathcal{D}^{!}}$. To see that $\lambda_{F}^{!}$is well-defined, suppose that $\phi_{1} \hat{v}_{1}=\phi_{2} \hat{v}_{2}$ with $\phi_{1}, \phi_{2} \in F$ and $\hat{v}_{1}, \hat{v}_{2} \in V_{\mathbb{C}[\mathcal{D}]}$. Choose $\alpha \in S$ such that $\alpha \phi_{1}, \alpha \phi_{2} \in \mathbb{C}[\mathcal{D}]$. Then $\alpha \phi_{1} \hat{v}_{1}=\alpha \phi_{2} \hat{v}_{2}, \lambda^{!}\left(\alpha \phi_{1} \hat{v}_{1}\right)=\lambda^{!}\left(\alpha \phi_{2} \hat{v}_{2}\right)$, and therefore $\alpha \phi_{1} \lambda^{!}\left(\hat{v}_{1}\right)=\alpha \phi_{2} \lambda^{!}\left(\hat{v}_{2}\right)$. Consequently, $\phi_{1} \lambda^{!}\left(\hat{v}_{1}\right)=\phi_{2} \lambda^{!}\left(\hat{v}_{2}\right)$, and this shows that $\lambda !$ is well-defined on $V_{F}$.

Now for every $r \in \mathcal{R}$ :

$$
\lambda_{F}^{!}\left(\hat{v}_{r}\right)(d)=\lambda_{d}\left(\xi_{d}\left(\hat{v}_{r}\right)\right)=\lambda_{d}\left(v_{r, d}\right)=x_{r, d}, \quad \forall d \in \mathcal{D}^{!},
$$

thus $\lambda_{F}^{!}\left(\hat{v}_{r}\right)=x_{r}$ lies in the subset $\mathbb{C}[\mathcal{D}]$ of $\mathbb{C}^{\mathcal{D}^{!}}$. From the proof of Bernstein's theorem, we know that the $F$-linear span of $\left\{\hat{v}_{r} \mid r \in \mathcal{R}\right\}$ is all of $V_{F}$, hence the image of $\lambda_{F}^{!}$is contained in $F \subset \mathbb{C}_{\text {loc }}^{\mathcal{D}^{!}}$. It follows that $\lambda:=\lambda_{F}^{!}$is the unique solution to $\Xi$ in $V_{F}^{*}$. Now let $\hat{v} \in V_{\mathbb{C}[\mathcal{D}]}$. Then $\lambda(\hat{v})=\lambda^{!}(\hat{v})$ lies in $\mathbb{C}^{\mathcal{D}^{!}}$by construction, and $\lambda(\hat{v}) \in F$, hence $\lambda(\hat{v}) \in \mathbb{C}^{\mathcal{D}^{!}} \cap F=F^{!}$. Thus $\lambda: V_{\mathbb{C}[\mathcal{D}]} \rightarrow F^{!}$ as asserted. If $\mathcal{D}^{!}=\mathcal{D}$, then $F^{!}=\mathbb{C}[\mathcal{D}]$, and the last statement of the corollary follows.

\section{Analytic Continuation of Whittaker Functionals}

As an application of Bernstein's theorem and the corollary of Section 1, we prove that the Whittaker functionals for induced representations of the metaplectic group possess an analytic continuation with no singularities. 
Let $\mathbb{F}$ be a nonarchimedean local field such that the group $\mu_{n}$ of $n$-th roots of unity in $\mathbb{F}^{\times}$has cardinality $n \geq 1$. We also assume that $|n|_{\mathbb{F}}=1$. Let $G:=G L_{r}(\mathbb{F}), r \geq 1$, and let $\widetilde{G}$ be the $n$-fold metaplectic cover of $G$. As a set, $\widetilde{G}:=G \times \mu_{n}$, with a multiplication law given by:

$$
(g, \zeta)\left(g^{\prime}, \zeta^{\prime}\right)=\left(g g^{\prime}, \zeta \zeta^{\prime} \sigma_{r}\left(g, g^{\prime}\right)\right), \quad \forall g, g^{\prime} \in G, \zeta, \zeta^{\prime} \in \mu_{n} .
$$

Here $\sigma_{r}: G \times G \rightarrow \mu_{n}$ is a 2-cocycle in $Z^{2}\left(G ; \mu_{n}\right)$ constructed in [2] from the Steinberg symbol $c:=(\cdot, \cdot)_{\mathbb{F}}^{-1}$, where $(\cdot, \cdot)_{\mathbb{F}}: \mathbb{F}^{\times} \times \mathbb{F}^{\times} \rightarrow \mu_{n}$ is the $n$-th order Hilbert symbol on $\mathbb{F}$. The 2-cocycle $\sigma_{r}$ can be explicitly computed on $G \times G$. In particular, for elements of $T$, the subgroup of diagonal matrices in $G$, we have:

$$
\sigma_{r}\left(t, t^{\prime}\right)=\prod_{1 \leq i<j \leq r}\left(t_{i}, t_{j}^{\prime}\right)_{\mathbb{F}}, \quad \forall t=\operatorname{diag}\left(t_{1}, \ldots, t_{r}\right), t^{\prime}=\operatorname{diag}\left(t_{1}^{\prime}, \ldots, t_{r}^{\prime}\right) \in T .
$$

We identify $\mu_{n}$ with its image under the embedding $\mu_{n} \hookrightarrow \widetilde{G}, \zeta \mapsto(e, \zeta)$, where $e$ is the identity in $G$. Let $\mathbf{p}: \widetilde{G} \rightarrow G$ be the projection homomorphism $(g, \zeta) \mapsto g$. Then the sequence:

$$
1 \rightarrow \mu_{n} \hookrightarrow \widetilde{G} \stackrel{\mathbf{p}}{\longrightarrow} G \rightarrow 1
$$

is exact. For any subgroup $H$ of $G$, we will denote by $\widetilde{H}$ the full metaplectic preimage $\mathbf{p}^{-1}(H)$ in $\widetilde{G}$. Finally, let $\mathbf{s}: G \rightarrow \widetilde{G}$ be the $\mathbf{p}$-section $g \mapsto(g, 1)$. Note that $\mathbf{s}$ is not a homomorphism if $r \geq 2$ since $\mathbf{s}(g) \mathbf{s}\left(g^{\prime}\right)=\mathbf{s}\left(g g^{\prime}\right) \sigma_{r}\left(g, g^{\prime}\right)$ for all $g, g^{\prime} \in G$.

The cover $\widetilde{G}$ splits canonically over $N$, the standard subgroup of upper triangular unipotent matrices in $G$. In fact, the restriction of $\mathbf{s}$ to $N$ yields the embedding $N \hookrightarrow \widetilde{G}$, and we denote by $N^{\mathbf{s}}$ its image $\mathbf{s}(N)$. Let $\psi_{\mathbb{F}}: \mathbb{F} \rightarrow \mathbb{C}^{\times}$ be a fixed nontrivial additive character, and let $\psi: N^{\mathbf{s}} \rightarrow \mathbb{C}^{\times}$be the standard nondegenerate character given by:

$$
\psi(\mathbf{s}(n)):=\prod_{i=1}^{r-1} \psi_{\mathbb{F}}\left(n_{i, i+1}\right), \quad \forall n \in N
$$

If $H$ is any subgroup of $G$, and $\pi$ is a representation of $\widetilde{H}$ on a complex vector space $V$, then a $\psi$-Whittaker functional for $\pi$ is a linear functional $\lambda: V \rightarrow \mathbb{C}$ such that $\lambda(\pi(n) v)=\psi(n) \lambda(v)$ for all $n \in N^{\mathbf{s}} \cap \widetilde{H}, v \in V$. We denote by $\mathrm{Wh}(\pi ; \psi)$ the space of all such functionals.

Since $|n|_{\mathbb{F}}=1$, the cover $\widetilde{G}$ also splits canonically over the maximal compact subgroup $K:=G L_{r}(\mathcal{O})$, where $\mathcal{O}$ is the ring of integers in $\mathbb{F}$ (cf. $\S 0.1$ of [6]). Let $\mathbf{k}: K \hookrightarrow \widetilde{G}$ be the canonical splitting homomorphism, and let $K^{\mathbf{k}}:=\mathbf{k}(K)$. For every $m \geq 0$, let $K_{m}:=\left\{k \in K \mid k \equiv e\left(\bmod \wp^{m}\right)\right\}$, where $\wp$ is the unique maximal ideal in $\mathcal{O}$, and let $K_{m}^{\mathbf{k}}:=\mathbf{k}\left(K_{m}\right)$. The collection $\left\{K_{m}^{\mathbf{k}} \mid m \geq 0\right\}$ is a basis of compact open neighborhoods of the identity $\tilde{e}$ in $\widetilde{G}$. 
Let $r_{1}, \ldots, r_{k}$ be positive integers such that $r=r_{1}+\ldots+r_{k}$, and let $M$ be the standard Levi subgroup in $G$ of type $\left(r_{1}, \ldots, r_{k}\right)$. Then $M$ is isomorphic to $G L_{r_{1}}(\mathbb{F}) \times \ldots \times G L_{r_{k}}(\mathbb{F})$, where the $G L_{r_{i}}(\mathbb{F})$ 's are embedded in $G$ as blocks along the diagonal. For every $s=\left(s_{1}, \ldots, s_{k}\right) \in \mathbb{C}^{k}$, let $\delta^{s}: M \rightarrow \mathbb{C}^{\times}$be the unramified quasicharacter given by:

$$
\delta^{s}(m):=\prod_{i=1}^{k}\left|\operatorname{det}\left(m_{i}\right)\right|_{\mathbb{F}}^{s_{i}}, \quad \forall m=\operatorname{diag}\left(m_{1}, \ldots, m_{k}\right) \in M .
$$

We will also regard $\delta^{s}$ as a quasicharacter of $\widetilde{M}$ that is trivial on $\mu_{n}$. Let $P$ be the standard parabolic subgroup in $G$ of type $\left(r_{1}, \ldots, r_{k}\right), U$ its unipotent radical, and $U^{\mathbf{s}}:=\mathbf{s}(U)$.

Now let $\pi: \widetilde{M} \rightarrow \operatorname{Aut}(V)$ be an admissible representation such that the dimension $\ell$ of $\mathrm{Wh}(\pi ; \psi)$ is nonzero (by theorem 1.5.2 of [6], $\ell<\infty$ ). Such a representation is said to be generic. For all $s \in \mathbb{C}^{k}$, let $\delta^{s} \pi: \widetilde{M} \rightarrow \operatorname{Aut}(V)$ be the admissible representation given by $\delta^{s} \pi(m):=\delta^{s}(m) \pi(m)$ for all $m \in \widetilde{M}$. It is easily shown that $\operatorname{dim}\left(\mathrm{Wh}\left(\delta^{s} \pi ; \psi\right)\right)=\ell$ for all $s \in \mathbb{C}^{k}$. Extend each $\delta^{s} \pi$ to a representation of $\widetilde{P}$ by letting $U^{\mathbf{s}}$ act trivially, and let $\pi_{s}$ be the unnormalized induced representation $\operatorname{Ind}\left(\widetilde{P}, \widetilde{G} ; \delta^{s} \pi\right)$. In other words, $\pi_{s}$ is the representation obtained from the action of $\widetilde{G}$ by right translation on the vector space:

$$
V_{s}:=\left\{f_{s} \in C^{\infty}(\widetilde{G} ; V) \mid f_{s}(p g)=\delta^{s} \pi(p) f_{s}(g), \quad \forall p \in \widetilde{P}, g \in \widetilde{G}\right\},
$$

where $C^{\infty}(\widetilde{G} ; V)$ is the space of locally-constant functions $f: \widetilde{G} \rightarrow V$. According to the results of $[1], \operatorname{dim}\left(\mathrm{Wh}\left(\pi_{s} ; \psi\right)\right)=\ell$ for all $s \in \mathbb{C}^{k}$.

Lemma 1. Let $\mathcal{V}$ be the space of functions $f \in C^{\infty}\left(K^{\mathbf{k}} ; V\right)$ that satisfy:

$$
f(p k)=\pi(p) f(k), \quad \forall p \in \widetilde{P} \cap K^{\mathbf{k}}, k \in K^{\mathbf{k}} .
$$

Then the map $V_{s} \rightarrow \mathcal{V},\left.f_{s} \mapsto f_{s}\right|_{K^{\mathbf{k}}}$, is an isomorphism for every $s \in \mathbb{C}^{k}$.

Proof. The proof is straightforward in view of the fact that $\widetilde{G}=\widetilde{P} K^{\mathbf{k}}$.

An element $f \in \mathcal{V}$ is called a flat section of the series $\left\{\pi_{s} \mid s \in \mathbb{C}^{k}\right\}$. By the lemma, $\mathcal{V}$ can be regarded as the space of $\pi_{s}$ for every $s \in \mathbb{C}^{k}$. By abuse of notation we define $\pi_{s}: \widetilde{G} \rightarrow \operatorname{Aut}(\mathcal{V})$ so that the diagram:

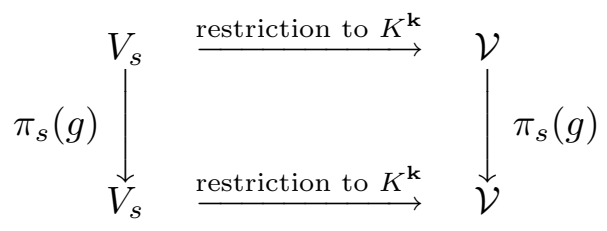

commutes for all $g \in \widetilde{G}$. 
Lemma 2. For all $g \in \widetilde{G}$ and $f \in \mathcal{V}$, the function $\mathbb{C}^{k} \rightarrow \mathcal{V}, s \mapsto \pi_{s}(g) f$, is regular in the sense of $\S 1$.

Proof. This is well-known and follows from the fact that $s \mapsto \delta^{s}(p)$ is regular for any $p \in \widetilde{P}$.

The main result of this section is the following theorem, which describes the analytic continuation of the $\psi$-Whittaker functionals for the series $\left\{\pi_{s} \mid s \in \mathbb{C}^{k}\right\}$. Theorem. For all $1 \leq i \leq \ell$ and $s \in \mathbb{C}^{k}$, one can choose $\lambda_{i, s} \in \mathrm{Wh}\left(\pi_{s} ; \psi\right)$ so that:

(a) For each $s \in \mathbb{C}^{k}$, the collection $\left\{\lambda_{i, s} \mid 1 \leq i \leq \ell\right\}$ is a basis for $\mathrm{Wh}\left(\pi_{s} ; \psi\right)$,

(b) For every $f \in \mathcal{V}$ and $1 \leq i \leq \ell$, the map $\mathbb{C}^{k} \rightarrow \mathbb{C}, s \mapsto \lambda_{i, s}(f)$, is holomorphic.

The proof is a straightforward application of the corollary to Bernstein's theorem in Section 1. Before turning to the proof, we will establish some preliminary results of a technical nature.

For the rest of this section, let $W\left[\right.$ resp. $\left.W_{M}\right]$ be the Weyl group of permutation matrices in $G$ [resp. M], and let $w$ be the long element in $\left[W / W_{M}\right]$ (cf. [4]). Then $M_{w}:=w M w^{-1}$ is the standard Levi subgroup in $G$ of type $\left(r_{k}, \ldots, r_{1}\right)$. Let $P_{w}$ be the standard parabolic subgroup in $G$ of type $\left(r_{k}, \ldots, r_{1}\right), U_{w}$ its unipotent radical, and $U_{w}^{\mathbf{s}}:=\mathbf{s}\left(U_{w}\right)$. Finally, let $\tilde{w}:=\mathbf{s}(w)$.

Lemma 3. For every $m>0, \tilde{w}^{-1} U_{w}^{\mathbf{s}} \cap \widetilde{P} \tilde{w}^{-1} K_{m}^{\mathbf{k}}=\tilde{w}^{-1}\left(U_{w}^{\mathbf{s}} \cap K_{m}^{\mathbf{k}}\right)$.

Proof. Since $\left.\mathbf{k}\right|_{N \cap K}=\left.\mathbf{s}\right|_{N \cap K}$ (cf. $\S 0.1$ of [6]), this follows from the corresponding nonmetaplectic statement:

$$
w^{-1} U_{w} \cap P w^{-1} K_{m}=w^{-1}\left(U_{w} \cap K_{m}\right),
$$

which is easily shown.

We now introduce some special functions in $\mathcal{V}$ to be used below. First, let:

$$
\check{f}_{n, f, s}:=\pi_{s}(n) f-\psi(n) f, \quad \forall n \in N^{\mathbf{s}}, f \in \mathcal{V}, s \in \mathbb{C}^{k} .
$$

Next, for any $v \in V$, choose $m \gg 0$ so that $v$ is fixed by $\widetilde{P} \cap K_{m}^{\mathbf{k}}$. Then for all $k \in K^{\mathbf{k}}$, let:

$$
\check{f}_{v, m}(k):= \begin{cases}\pi(p) v & \text { if } g=p \tilde{w}^{-1} k^{\prime} \text { for some } p \in \widetilde{P} \cap K^{\mathbf{k}}, k^{\prime} \in K_{m}^{\mathbf{k}}, \\ 0 & \text { otherwise. }\end{cases}
$$

Then $\check{f}_{v, m}$ is a well-defined element of the space $\mathcal{V}^{m}$ of $K_{m}^{\mathbf{k}}$-fixed vectors in $\mathcal{V}$. 
Proposition. Let $\left\{\lambda_{i} \mid 1 \leq i \leq \ell\right\}$ be a fixed basis for $\mathrm{Wh}(\pi ; \psi)$. Then:

(a) For any compact open subgroup $\mathcal{C} \subset U_{w}$, let $\mathcal{C} \mathbf{s}:=\mathbf{s}(\mathcal{C})$, and let:

$$
\lambda_{i, s}^{\mathcal{C}}(f):=\int_{\mathcal{C}^{\mathbf{s}}} \lambda_{i}\left(\pi_{s}\left(\tilde{w}^{-1} u\right) f(\tilde{e})\right) \bar{\psi}(u) d u, \quad \forall 1 \leq i \leq \ell, s \in \mathbb{C}^{k}, f \in \mathcal{V},
$$

where $d u$ is a left Haar measure for $U_{w}^{\mathbf{s}}$. If $\mathcal{C}$ is sufficiently large (depending only on $f$ ), then $\lambda_{i, s}(f):=\lambda_{i, s}^{\mathcal{C}}(f)$ is independent of $\mathcal{C}$,

(b) For all $s \in \mathbb{C}^{k}$, the collection $\left\{\lambda_{i, s} \mid 1 \leq i \leq \ell\right\}$ is a basis for $\operatorname{Wh}\left(\pi_{s} ; \psi\right)$,

(c) If $\left\{v_{j} \mid 1 \leq j \leq \ell\right\}$ are elements of $V$ such that $\lambda_{i}\left(v_{j}\right)=\delta_{i j}$, and $m$ is a sufficiently large positive integer, then for all $s \in \mathbb{C}^{k}$ :

$$
\mathcal{V}=\operatorname{span}\left\langle\check{f}_{v_{j}, m} \mid 1 \leq j \leq \ell\right\rangle \oplus \operatorname{span}\left\langle\check{f}_{n, f, s} \mid n \in N^{\mathbf{s}}, f \in \mathcal{V}\right\rangle .
$$

Proof. Let $s \in \mathbb{C}^{k}$ be fixed throughout the proof, and let:

$$
\mathcal{V}_{\psi, s}:=\operatorname{span}\left\langle\check{f}_{n, f, s} \mid n \in N^{\mathbf{s}}, f \in \mathcal{V}\right\rangle .
$$

Since $\check{f}_{n, f, s}:=\pi_{s}(n) f-\psi(n) f$, a linear functional $\lambda: \mathcal{V} \rightarrow \mathbb{C}$ lies in $\operatorname{Wh}\left(\pi_{s} ; \psi\right)$ if and only if $\mathcal{V}_{\psi, s} \subset \operatorname{ker}(\lambda)$. Consequently, $\operatorname{Wh}\left(\pi_{s} ; \psi\right)$ can be identified with the linear dual of $\mathcal{V} / \mathcal{V}_{\psi, s}$, hence $\operatorname{dim}\left(\mathcal{V} / \mathcal{V}_{\psi, s}\right)=\ell$. Thus, to prove (c) it suffices to show that the $\ell$ elements $\left\{\check{f}_{v_{j}, m}\right\}$ are linearly independent and not contained in $\mathcal{V}_{\psi, s}$.

First, we claim that if $\check{f} \in \mathcal{V}_{\psi, s}$, then $\lambda_{i, s}^{\mathcal{C}}(\check{f})=0$ if $\mathcal{C}$ is sufficiently large. It suffices to prove this for elements of the form $\check{f}_{n, f, s}$, where $n \in N^{\mathbf{s}}$ and $f \in \mathcal{V}$. Write $n=n^{\prime} u^{\prime}$ with $n^{\prime} \in N_{w}^{\mathbf{s}}:=N^{\mathbf{s}} \cap \widetilde{M}_{w}$ and $u^{\prime} \in U_{w}^{\mathbf{s}}$. Let $\mathcal{C}$ be any compact open subgroup of $U_{w}$ such that $u^{\prime} \in \mathcal{C}^{\mathbf{s}}$, and $n^{\prime} \mathcal{C}^{\mathbf{s}} n^{\prime-1}=\mathcal{C}^{\mathbf{s}}$. Then:

$$
\lambda_{i, s}^{\mathcal{C}}\left(\pi_{s}(n) f\right)=\int_{\mathcal{C}^{\mathbf{s}}} \lambda_{i}\left(\pi_{s}\left(\tilde{w}^{-1} u n\right) f(\tilde{e})\right) \bar{\psi}(u) d u=\int_{\mathcal{C}^{\mathbf{s}}} \lambda_{i}\left(f_{s}\left(\tilde{w}^{-1} u n^{\prime} u^{\prime}\right)\right) \bar{\psi}(u) d u
$$

where $f_{s} \in V_{s}$ corresponds to $f$ under the isomorphism $V_{s} \cong \mathcal{V}$ of lemma 1 . After a change of variables $\left\{u \mapsto n^{\prime} u n^{\prime-1} ; d u \mapsto d u\right\}$, we obtain:

$$
\begin{gathered}
\int_{\mathcal{C}^{\mathbf{s}}} \lambda_{i}\left(f_{s}\left(\tilde{w}^{-1} n^{\prime} u u^{\prime}\right)\right) \bar{\psi}\left(n^{\prime} u n^{\prime-1}\right) d u=\int_{\mathcal{C}^{\mathbf{s}}} \lambda_{i}\left(\pi\left(\tilde{w}^{-1} n^{\prime} \tilde{w}\right) f_{s}\left(\tilde{w}^{-1} u u^{\prime}\right)\right) \bar{\psi}(u) d u \\
=\psi\left(\tilde{w}^{-1} n^{\prime} \tilde{w}\right) \int_{\mathcal{C}^{\mathbf{s}}} \lambda_{i}\left(f_{s}\left(w^{-1} u u^{\prime}\right)\right) \bar{\psi}(u) d u .
\end{gathered}
$$

Note that $\psi\left(\tilde{w}^{-1} n^{\prime} \tilde{w}\right)=\psi\left(n^{\prime}\right)$ for all $n^{\prime} \in N_{w}^{\mathbf{s}}$, since conjugation by $\tilde{w}$ permutes the blocks of $N_{w}^{\mathbf{s}}$. After a change of variables $\left\{u \mapsto u u^{\prime-1} ; d u \mapsto d u\right\}$, the preceding expression becomes: 


$$
\begin{aligned}
\psi\left(n^{\prime}\right) \int_{\mathcal{C}^{\mathbf{s}}} \lambda_{i}\left(f_{s}\left(\tilde{w}^{-1} u\right)\right) \bar{\psi}\left(u u^{\prime-1}\right) d u & =\psi(n) \int_{\mathcal{C}^{\mathbf{s}}} \lambda_{i}\left(\pi_{s}\left(\tilde{w}^{-1} u\right) f(\tilde{e})\right) \bar{\psi}(u) d u \\
& =\psi(n) \lambda_{i, s}^{\mathcal{C}}(f),
\end{aligned}
$$

since $\bar{\psi}\left(u^{\prime-1}\right)=\psi\left(u^{\prime}\right)$, and $\psi\left(n^{\prime}\right) \psi\left(u^{\prime}\right)=\psi\left(n^{\prime} u^{\prime}\right)=\psi(n)$. We have therefore shown that $\lambda_{i, s}^{\mathcal{C}}\left(\pi_{s}(n) f\right)=\psi(n) \lambda_{i, s}^{\mathcal{C}}(f)$, or that $\lambda_{i, s}^{\mathcal{C}}\left(\check{f}_{n, f, s}\right)=0$, provided that $\mathcal{C}$ is sufficiently large. This establishes the claim.

Next, let $\left\{v_{j} \mid 1 \leq j \leq \ell\right\}$ be elements of $V$ such that $\lambda_{i}\left(v_{j}\right)=\delta_{i j}$. Choose $m \gg 0$ so that each $v_{j}$ is fixed by $\widetilde{P} \cap K_{m}^{\mathbf{k}}$, and $\left.\psi\right|_{N^{\mathbf{s}} \cap K_{m}^{\mathbf{k}}}=1$. Then for any compact open subgroup $\mathcal{C} \subset U_{w}$ containing $U_{w} \cap K_{m}$ :

$$
\lambda_{i, s}^{\mathcal{C}}\left(\check{f}_{v_{j}, m}\right)=\int_{\mathcal{C}^{\mathbf{s}}} \lambda_{i}\left(\pi_{s}\left(\tilde{w}^{-1} u\right) \check{f}_{v_{j}, m}(\tilde{e})\right) \bar{\psi}(u) d u=\int_{\mathcal{C}^{\mathbf{s}}} \lambda_{i}\left(f_{s}\left(\tilde{w}^{-1} u\right)\right) \bar{\psi}(u) d u
$$

where $f_{s} \in V_{s}$ now corresponds to $\check{f}_{v_{j}, m}$ under the isomorphism of lemma 1 . Since $f_{s}$ is supported on $\widetilde{P} \tilde{w}^{-1} K_{m}^{\mathbf{k}}$, lemma 3 implies that:

$$
\lambda_{i, s}^{\mathcal{C}}\left(\check{f}_{v_{j}, m}\right)=\int_{U_{w}^{\mathbf{s}} \cap K_{m}^{\mathbf{k}}} \lambda_{i}\left(\check{f}_{v_{j}, m}\left(\tilde{w}^{-1} u\right)\right) \bar{\psi}(u) d u=c_{m} \delta_{i j},
$$

where $c_{m}$ is the volume of $U_{w}^{\mathbf{s}} \cap K_{m}^{\mathbf{k}}$ with respect to the measure $d u$. Since $c_{m} \neq 0$, the elements $\left\{\check{f}_{v_{j}, m} \mid 1 \leq j \leq \ell\right\}$ are linearly independent and not contained in $\mathcal{V}_{\psi, s}$, and (c) is proved. The other statements follow easily from (c) and the preceding calculations.

Proof of Theorem: Part (a) of the theorem has already been proved. We continue to use the notation of the proposition. Let $1 \leq i \leq \ell$ be fixed, let $\mathcal{D}:=\mathbb{C}^{k}$, and let $\mathcal{R}$ be the indexing set:

$$
\mathcal{R}:=\left\{(n, f) \mid n \in N^{\mathbf{s}}, f \in \mathcal{V}\right\} \cup\{(j) \mid 1 \leq j \leq \ell\} .
$$

For $r=(n, f)$ and $s \in \mathcal{D}$, let $v_{r, s}:=\check{f}_{n, f, s}$, and let $x_{r, s}:=0$. For $r=(j)$ and $s \in \mathcal{D}$, let $v_{r, s}:=\check{f}_{v_{j}, m}$, and let $x_{r, s}:=c_{m} \delta_{i j}$. Let:

$$
\Xi_{s}:=\left\{\left(v_{r, s}, x_{r, s}\right) \in \mathcal{V} \times \mathbb{C} \mid r \in \mathcal{R}\right\}, \quad \forall s \in \mathbb{C}^{k} .
$$

By lemma 2, the collection $\Xi_{\mathcal{D}}:=\left\{\Xi_{s} \mid s \in \mathcal{D}\right\}$ is a regular family in the sense of Section 1. By part (c) of the proposition, it follows that $\lambda_{i, s}$ is the unique solution to $\Xi_{s}$ for all $s \in \mathbb{C}^{k}$. Since $\mathcal{V}=\bigcup_{m \geq 0} \mathcal{V}^{m}$ has countable dimension over $\mathbb{C}$, we see that all of the hypotheses of Bernstein's theorem and the corollary are met. Thus, if $\lambda$ is the unique solution to $\Xi$, then $\lambda: \mathcal{V}_{\mathbb{C}[\mathcal{D}]} \rightarrow \mathbb{C}[\mathcal{D}]$. In particular, for all $f \in \mathcal{V}$, the map $\mathbb{C}^{k} \rightarrow \mathbb{C}, s \mapsto \lambda_{i, s}(f)$, is none other than $\lambda(f \otimes 1) \in \mathbb{C}[\mathcal{D}]$, which proves part (b) of the theorem. 


\section{References}

[1] W. Banks, Heredity of Whittaker models on the metaplectic group, Pacific J. Math 185 (1998), 89-96.

[2] W. Banks, J. Levy, and M. Sepanski, Block-compatible metaplectic cocycles, J. Reine Angew. Math., to appear.

[3] I. Bernstein, Letter to I. Piatetski-Shapiro.

[4] W. Casselman, Introduction to the theory of admissible representations of p-adic reductive groups, Lecture notes.

[5] S. Friedberg and D. Goldberg, On local coefficients for non-generic representations of some classical groups, Compositio Math., to appear.

[6] D. Kazhdan and S. Patterson, Metaplectic forms, Inst. Hautes Ètudes Sci. Publ. Math. 59 (1984), 35-142.

Mathematics Department, University of Missouri - Columbia, 202 Mathematical Sciences Bldg., Columbia, MO 65211

E-mail address: bbanks@math.missouri.edu 